\title{
Review on water quality monitoring technologies
}

\author{
N. T. J. Ong, S. K. Yee \\ Research Center for Applied Electromagnetic, Faculty of Electrical and Electronic Engineering, \\ Universiti Tun Hussein Onn Malaysia, Malaysia
}

\begin{tabular}{l}
\hline \hline Article Info \\
\hline Article history: \\
Received Jul 27, 2019 \\
Revised Oct 29, 2019 \\
Accepted Nov 13, 2019 \\
\hline
\end{tabular}

Keywords:

Contaminant

Conventional technique

Microwave technique

Monitoring

Water quality

\begin{abstract}
Water quality monitoring is always the prior element to ensure the drinking water is safe to be consumed. A lot of researches have been carried out over the past decades to design and develop a robust and cost-effective water monitoring system. The conventional water monitoring techniques were based on laboratory instruments which is time-consuming and laborious. Furthermore, it is not suitable when the water sampling point is far from the commercial laboratory centres. These limitations were then been solved by the developments of portable testing kits and microwave technique. The microwave techniques such as spectroscopy techniques and microwave sensor approach have improved the water quality monitoring experience which is convenient without sacrificing the measurement accuracy and sensitivity. Its portability enables the on-site measurement at rural areas and thus reduce the transportation and manpower cost. This paper intends to review the water contaminant detection techniques which include standardized drinking water parameter testing and microwave-based in terms of physical, chemical and microbiological parameters. Furthermore, this review also emphasizes the current trend of the water quality testing method in microwave technique. At the end of this paper, a significant advantages and drawbacks of the techniques are summarized, and recommendations are provided for future development in the water quality monitoring.
\end{abstract}

Copyright $(2020$ Institute of Advanced Engineering and Science. All rights reserved.

\section{Corresponding Author:}

Josephine Ong Ning Ting,

Research Center for Applied Electromagnetic,

Faculty of Electrical and Electronic Engineering,

Universiti Tun Hussein Onn Malaysia, 86400 Parit Raja, Batu Pahat Johor, Malaysia.

Email: josephineong94_@hotmail.com

\section{INTRODUCTION}

Water is one of the essential elements for life on earth. In Malaysia, 99\% of the water supply is mainly from surface water whereas another $1 \%$ of them came from underground [1]. According to the Environmental Quality Report 2017, the renewable water and total internal water resources that have been received by Malaysia per capita annually from its extensive river system was about $25 \mathrm{~km}^{3}$ and $580 \mathrm{~km}^{3}$ respectively, where $30 \%$ of them were used for municipal uses [1,2]. All the surface water and groundwater were required to be treated before distributing to the consumers. In Malaysia, there were about 488 water treatment plant (WTP) have been established to treat the municipal water and they are technically under the jurisdiction of the local state governments [1]. However, some of them still provide a conventional water treatment system which is no longer capable to remove the emerging contaminants in water supply as the rapid growth in population and industrial activities has seriously deteriorate the quality of the water resources $[1,2]$.

The factors that resulting the contamination in drinking water include the internal corrosion of pipeline, unhygienic drinking water practice, and anthropogenic activities. In past, Malaysia has implemented Galvanized Iron (GI) pipe, however, due to the internal corrosion of the GI pipe, it is then being replaced by 
the pipe made from plastic, Stainless Steel (SS), Ductile Iron (DI) and Mild Steel (MS) [1]. Nevertheless, the risk of the internal corrosion of GI pipeline still expose to human life in nowadays because of insufficient budgets and some of them were still under a good condition. Although the water treatment plants have been established to reduce the concentration of heavy metals, however, the heavy metals may still expose to human as the result of chemical substances used for water treatment process. N. H. Ab Razak et al stated that the chemical such as Alum (Al) that has been used extensively in conventional water treatment system had become the source of the water contamination as well [3]. In Malaysia, many households had installed the water filter system in their house, however, the insufficient sanitation and poor hygiene handling such as improper drinking water storage container and unhygienic handlers will become a transport medium for contaiminamts to enter into drinking water [1]. For an example, the filtration unit which is not maintained properly will result in the leaching of heavy metals such as Aluminium ( $\mathrm{Al}$ ), Chromium $(\mathrm{Cr})$ and Nickel (Ni) from $\mathrm{Al}$ and SS containers into the drinking water [3]. Therefore, the water quality assessment has become the prior element and should be carried out periodically to ensure the drinking water is safe to be consumed [4].

Recently, there are a wide variety of water contaminant detection technique such as sample-based method and microwave sensor-based method [5]. Sample-based water contaminant detection method has been introduced since 1917s which involved the use of international standard laboratory instruments in a laboratory that manually handled by a group of trained and skillful technicians or laboratory assistants [5]. The conventional laboratory techniques usually involved laboratory and portable testing kits. Microwave sensor-based method involved the use of smart sensor to monitor water quality [6]. This model has been adopted in various water contaminants detection which include water quality sensor, microfluidic sensors and spectroscopy technique [7]. Water quality sensor has been introduced at the early of 1900s. It is suitable when the resources are limited as it required less time, simple and low-cost testing method. Furthermore, water quality sensor with simple testing methods enable the on-site water testing without sending the samples to the commercial laboratories [8]. Despite its popularity, however, microwave sensor has its downsides as it is less precise and accurate compared to the lab-based method. Hence, the laboratory assistants and other professionals will need to consider the sensitivity and accuracy of the selected microwave sensor during water testing. An overview in terms of physical, chemical and microbiological parameters in water quality monitoring will be discussed in this paper. This review also emphasizes the current trend of the water quality testing method based on microwave technique. Significant advantages and drawbacks of the techniques are discussed, and recommendations are provided for future development in the water quality monitoring.

\section{STANDARDIZED DRINKING WATER QUALITY TESTING}

In sub section 2.1, 2.2 and 2.3 in Section 2, the testing methods of drinking water will be categorized based on the characteristic of water in terms of physical, chemical and microbiological. A general working principal and operation of each type of testing method will be discussed and some of the related instruments that have been standardized and still available on market will also be discussed in the following sections.

\subsection{Physical Parameters Testing Methods}

Physical parameters can be determined by using the human sense such as $\mathrm{pH}$, turbidity and non-metals which has the immediate response in water such as Chorine $(\mathrm{Cl})$, Bromine $(\mathrm{Br})$, Iodine (I), and Sulphur (S) [5]. According to WHO, physical parameters do not cause a direct effect to human health issues, however, its characteristic can be used as an indicator to indicate the risk of water contamination which may be harmful to human [5]. Generally, a good physical of drinking water should be low turbidity or clear, non-taste and odorless.

\subsubsection{Turbidity Tube \& Digital Turbidimeter}

Turbidity tube is a portable kit which is used to estimate NTU by visually. It is easy and practical to be used since it does not require any power supply and replacement parts [5]. Moreover, it can be upkeep easily by rinsing it with clean water after remove the dirt. The range of measurement of turbidity tube is from 0 to 2,000 NTU, however, there is a limitation because it is hard to read the level which is less than 10 NTU.

Digital turbidimeter is an alternative device which requires the power supply or battery. It provides a more accurate result compared to the turbidity tube as the result are shown in digital readings rather than visually observation [5]. The range of digital turbidimeter measurement is from 0 to $1,000 \mathrm{NTU}$. It can be used to measure the turbidity level less than 10 NTU which is hardly to be obtained by using a turbidity tube. Digital turbidimeter is relatively expensive and vulnerable to be damaged compared to turbidity tube. It needs to be stored in a dry and clean environment as a small portion of dust on the optics of turbidimeter will 
influence the result of the measurement. Moreover, the water sample vials are needed to be cleaned regularly to avoid the dirt, water droplets and scratches which may lead to the stray light interference.

\subsubsection{Amperometry \& Ion Selective Field Effect Transistor (ISFET)}

Amperometry is used to measure $\mathrm{pH}$ value of the water sample [9]. It is easy to be handled and operates based on the generation of hydrogen with a less noble metal. A galvanic cell will be formed and generates a current where the cell's current is depending on the $\mathrm{pH}$ value. However, the differences between the sample composition may cause a big error during the measurement. Thus, this method is not suitable for the concentrated acids and bases measurement [9]. K. Tirumalesh had proposed a new ion chromatography method to detect Bromide (Br-) and Nitrate (NO-3) in contaminated waters by using a low-capacity anion exchange column with amperometry and absorbance detection. A $3 \mathrm{mM}$ sodium hydroxide $(\mathrm{NaOH})$ eluent is used for analyte ions separation. The eluent is passed through amperometric detector and anion suppressor unit for UV detection in order to quantify the concentration of $\mathrm{Br}$ - and NO-3. The concentration of $\mathrm{Br}$ - can be defined based on the peak area in amperometry whereas the concentration of NO-3 is obtained by substracting the $\mathrm{Br}$ - from total peak area and calculate its response factor in UV detector. This method provides an accurate result with a 0.998 correlation coefficinets and the calculated NO-3 concentration is mateched with the actual concentration. Furthermore, this method can detect the concentration of these ions as low as $0.03 \mathrm{mg} / \mathrm{L}-1[10]$.

Ion selective field effect transistor (ISFET) is a relatively new method in $\mathrm{pH}$ value measurement [11]. It had implemented the same concept as Amperometry which uses the flow of current between the source and drain, generated hydrogen ions to measure the $\mathrm{pH}$ value. ISFET has an advantage over Amperometry as it is relatively small, but they are less stable and efficient in a longer using time.

\subsubsection{Inductive Coupled Plasma}

Inductive Coupled Plasma is used to analyze the presence of non-metals such as $\mathrm{Cl}, \mathrm{Br}$, I and $\mathrm{S}$ by breaking down the samples to the atomic stage and determine the concentration of metals via Atomic Absorption Spectrometer (AAS) or mass spectroscopy. Inductive coupled plasma can be categorized into two main types which include inductively coupled plasma mass spectrometry (ICP-MS) and inductively coupled plasma optical emission spectroscopy (ICP-OES). ICP-MS is a type of mass spectrometry which is highly sensitive to the metals and enable to differentiate isotopic speciation for ions of choice [12]. It has a good precision, low detection limits and short time-taken for isotopic analysis. ICP-OES involved the manipulating of trace element to determine the concentrations of different elements. In previous work, the extraction and digestion method for metals determination was proposed and compared by using ICP-OES and ICP-MS. Both the instruments showed a relatively good result, but the amount of digested carbon nanotubes is only $5 \mathrm{mg}$ [13].

\subsection{Chemical Parameters Testing Methods}

The natural chemical materials such as As, fluoride (F-), iron (Fe) and $\mathrm{Mn}$ are usually found in groundwater whereas nitrogen $(\mathrm{N})$ and pesticides are found in water as the result of human activities. Unlike microbiological contamination, chemical contaminations such as NO-3 in drinking water pose a health issues only after years of exposure according to its concentration and the length of exposure time [9].

\subsubsection{Chromatography}

Chromatography is a technique used for the separation of individual components in a complex mixture [14]. It is usually combined with the mass spectroscopy to determine the chemical contaminants in water samples [14]. Basically, the samples will be placed in a specific packing which contains a certain type of chemicals. When the samples are passing through, a detector which is located at the end of the channel will quantifies the concentration of the samples. There are two types of chromatography which are liquid chromatography and gas chromatography. Albishri et al. had detected five organophosphorus pesticides in the range of 0.01-0.1 $\mathrm{ng} / \mathrm{mL}$ of tap, well and lake water by using the combination of UV-based reversed phase liquid chromatography and liquid phase micro extraction. The types of pesticides have been investigated based on their ionic liquid (IL) type, IL volume, ionic strength, sonication and centrifugal time, temperature, and speed. This proposed technique had increased the efficiency of the extraction as much as 98\% if compared to the conventional liquid chromatography [15]. Gas chromatography have been combined with the nitrogen-phosphorus detector (NPD) to detect the concentration of pesticides in drinking water [16]. However, its detection sensitivity was not efficient as liquid chromatography due to the characteristic of pesticides which is non-volatile and high molar mass. 


\subsubsection{Colour Disc Comparator \& Colorimeter}

Colour Disc Comparator is a visual comparison method which uses tablet reagent and colour charts to determine the concentration of tested contaminants. It is accurate and portable. During the measurement, reagent is added into the apparatus and the wheel is tuned until the sample matches the references colour. The concentration of the tested contaminant will then display on a window. Colour disc comparator can test for a range of chemical parameters such as $\mathrm{Cl}, \mathrm{NO}-3, \mathrm{Fe}$ and $\mathrm{Mn}$. It is easier to handle and accurate compared to test strips, however, the cost for colour disc comparator is relatively high [5].

Colorimeter is a light-sensitive device which can be used to measure the transmittance and light absorbance that is passing through the water sample. During the measurement, the chemical reagent which react with the specific chemical parameters such as Fe and $\mathrm{Mn}$ will be added into the water sample and determine the concentration of the colour [17]. The most common colorimeter which is available on market is DR 900 Multiparameter Handheld Colorimeter. It is operated with AA batteries, which is portable and able to test about 90 types of chemical and physical parameters such as $\mathrm{Cl}, \mathrm{Fe}, \mathrm{pH}$, turbidity and more. It can store up to 500 data sets and enable file transferring by using a USB cable without any software conversion [5].

\subsubsection{Atomic Absorption Spectrometer (AAS)}

Atomic Absorption Spectrometer (AAS) is used to detect the presence of metals in water sample. The concentration of metal will be determined by light absorption of the metal atoms when the samples are heated by flame or electrically in a graphite furnace. In AAS, a hollow cathode lamp is used to generate the light source whereas photomultiplier tube is used as a detector. During the measurement, a monochromator will be used to distinguish the element light whereas the light source will be generated to reduce the unwanted radiation from detector [18]. There are two types of atomization methods which include flame atomization and electrothermal atomization. Flame AAS performed a continuous analytical signal whereas electrothermal AAS performed a discontinuous analytical signal which takes 2-4 minutes for per sample [19]. Flame AAS takes an advantage as the sample which is introduced into the spectrophotometer can be reproduced. However, the efficiency of the atomization is quite low due to the generous size of aerosol droplets produced during nebulization. It is hard to be carried to the flame and large amount of the combustion gases are needed to dilute the samples. The limitation of flame AAS was then been overcome by electrothermal AAS by trapping the gaseous analyte in a small graphite tube. Although the volume of the gaseous analyte is small, the resulting vapor phase is greater than in flame atomization. It provides a significant improvement in sensitivity and detection limitation. However, this technique has a limitation which the reproducibility of the sample is hard to manipulate as the atomization efficiency is depends on the samples in graphite tube.

\subsection{Microbiological Parameters Testing Methods}

Microorganisms are the prior issues in drinking water as the presence of microorganisms will causes a serious health problem to public. According to WHO, the water which is infected by human and animal feces brings a greatest risk to public health [9]. WHO Guidelines for Drinking Water Quality stated that the drinking water should not consists any fecal contaminant per $100 \mathrm{~mL}$ sample.

\subsubsection{Most Probable Number (MPN)}

Most probable number (MPN) is used to indicate the number of bacteria present in the water sample. A set of samples from $1 \mathrm{~mL}$ to $10 \mathrm{~mL}$ will be extracted from the same water samples will be added into a sterile tube and incubated at a specific temperature. The sterile tube is then being replaced with a disposable tray with multiple wells [20]. MPN is simple to be used with trays and it is popular for determine the E. coli on-site. The main limitation of using MPN is the results are statistical estimation and required more labour and training compared to P-A testing. Furthermore, the cost of this technique is high, and it is not practical when there is a large number of testing are needed as disposable trays will produce a lot of waste.

\subsubsection{Membrane Filtration}

Membrane filtration is internationally recognized by the USEPA and UNEP/ WHO and used to determine the number of bacteria in a water sample. It can be done either in laboratory or by using a portable test kit. The main function of membrane filtration is isolating and eliminating the microbiological organisms within a large number of sample volume [21]. During the measurement, a $100 \mathrm{~mL}$ water sample is vacuumed by using a small hand pump with filter paper, placed the remaining bacteria in a Petri dish with culture media after filtration, and placed the Petri dishes in an incubator at an appropriate temperature and time according to the type of indicator bacteria and culture media used. Finally, the number of colonies forming units (CFU) per $100 \mathrm{~mL}$ is counted based on the bacteria colonies [22]. The common standard of membrane filtration, 
selective medium and culture plate that used to detect the presence of E. coli and other microorganisms in drinking water was ISO 16654:2001 [22]. There are some limitations by using this technique such as laborious and time-consuming. The sensitivity for detecting contaminants at a low concentration is low due to differentiation in phenotypic traits is not consistent [23]. Moreover, it is difficult to test turbid water as the high turbidity will causes the clogging of the membrane and restricting the growth of indicator bacteria on the filter paper.

\section{MICROWAVE TECHNIQUE IN DRINKING WATER QUALITY TESTING}

The main challenge for water quality testing is detecting the water contaminants at low concentration continuously. The presence of contaminants in water will change the dielectric permittivity and conductivity of contaminants in water [24]. Hence, microwave techniques can determine the contaminants based on the contrast of dielectric constant between contaminated and clean water. The current microwave technique provides with an approximate estimation of the water compositions but does not fully occupied the needs of the users [24]. Therefore, a further research and development must be done to produce a high accuracy, low-cost and robust microwave sensor for water quality testing.

\subsection{Water Quality Sensor \& Microfluidic Sensor}

Water quality sensors has been used to detect and analyze some parameters such as $\mathrm{pH}$, turbidity and conductivity [25]. The commercial product of water quality sensors is currently available in the market and these sensors can be integrated and monitored by a single multiparameter controller [25]. A novel approach of real-time detection low-cost sensor network has been done by Lambrou et al with multiple electrochemical optical sensors to detect E. coli and As. The system consists of six sensors in-line which can detect conductivity, oxidation reduction potential (ORP), $\mathrm{pH}$, temperature, turbidity and water flow in the pipeline [26]. The overall system structure of the proposed sensor network consists of three main subsystems: central measurement node, control node and notification node. In first subsystem, a PIC32 MCU based board is used to integrate multiple sensors and collect their data before transmitting it to the other nodes. The control node, which is implemented by ARM/ Linux web-server based platform were used to store the measurement data which is received from central measurement node whereas the notification node with interconnected Zigbee RF transceiver is responsible to notified users via sms when the sensors detect a sudden change in water contaminants' concentration. A local indicator such as Buzzer, LCD and LED was also implemented as a local near-tap notification to alert the user when they are around the water contaminated area. The performance of the developed hardware was then been validated and evaluated by injecting various concentration of E. coli and As in the water sample. Results showed that during the measurement for E. coli, the sensors were capable to detect the concentrations of $0.05,0.5,5,5,000,50,000$ and 50,000,000 $\mathrm{CFU} / \mathrm{mL}$ whereas for As measurement, the sensors were not capable to detect the low concentration at 5 and $10 \mu \mathrm{g} / \mathrm{L}$. The $\mathrm{pH}$ and ORP sensors were then responded to the As at concentration of 25,50 and $125 \mu \mathrm{g} / \mathrm{L}$ whereas at the concentration of 500 and $1,000 \mu \mathrm{g} / \mathrm{L}$, all the sensors were responded well [26]. There are some limitations with the water quality sensor as some contaminants may not be detected due to the low sensitivity of the sensor at low concentration and the existence of new types of emerging contaminants [27]. Furthermore, the long transmission delays and slow response time of data capturing will cause the result to be inaccurate, the result may vary when there is changes in the contaminants' concentration and the sensor could not take an immediate response on this matter [7].

Microfluidic sensor is used to monitor or sense the microscale liquids [28]. It was initially developed from microelectrochemical systems (MEMS) technology but it is now being trained to discipline itself. Integrated microfluidic devices are frequently used for biological and chemical analysis. A work with low-cost and high sensitivity sensor has been developed by Jiang et al. for bacteria concentration determination. The concept of electrical impedance spectroscopy has been implemented in the design of sensor and it is able to detect concentration of 10 bacterial cells per mL based on microfluidic method [28]. Microfluidic sensor can be used to develop a cost-effective and high sensitivity commercial product. However, the pre-treatment of the sample must be concerned when it involves the use of lab-on-a-chip sensor to detect multiple contaminants because the overall process and system operation will become more complicated [29].

\subsection{Spectroscopy Techniques}

Spectroscopy technique is a microwave technique which involved the use of light electromagnetic radiation source and a specific probe to detect biological or chemical substances. There are three major types of spectroscopy techniques which Raman spectroscopy, Ground Penetrating Radar (GPR) and dielectric spectroscopy. 
Raman spectroscopy involved the excitation of atoms to a higher energy state and resulting in frequency shifting [30]. A research has been done to detect sulphate (SO2-4) ions and methane (CH4) in drinking water by using Raman Spectroscopy. This technique is simple to use, compactness, portability and sensitive to the changes in its environment. It can be used to detect the pesticides in food products and determine the chemical and biological composition in water. Raman spectroscopy tends to provide a robust testing result in water quality monitoring, however, the overlapping of absorption bands during spectrum analysis will weaken the spectral band and influence its efficiency on continuous on-line monitoring [31].

Ground Penetrating Radar (GPR) is the most common used instrument in pipeline water quality monitoring [32]. The general operation of GPR involved emitting a microwave electromagnetic (EM) radiation through the ground then returns to the ground surface. A work has been conducted by H. Abdelgwad [32] to detect water contaminants in underground pipelines. Result showed that there is a significant variation of reflection coefficient in dry soil compared to the moist soil as it will attenuate the reflected signal with the occurrence of water inside the pipeline. Furthermore, the concentrations of moisture and chloride (Cl-) ingress has been monitored and observed by using GPR amplitude attenuation through concrete cover [32]. In general, GPR is good at determining the areas where natural sedimentary layering is disturbed, however, it has some drawbacks as it is less efficiency when the terrain is not flat and even. Moreover, the accuracy and sensitivity of GPR is quite low when it is used to test in clay.

Carey and Hayzen has stated that the presence of contaminants in drinking water is correlated with the changes in dielectric constant [33]. These properties can be measured by using dielectric spectroscopy [33]. Dielectric spectroscopy takes advantages over conventional sensing techniques as it is robust and portable. The most known sensors such as Lubrigard® Dielectric Sensor has been developed based on dielectric measurement. There are three main types of dielectric properties measurement in water contaminants detection which include cavity perturbation, coaxial probe, and microstrip structure.

Microwave cavity resonator are popular to be used in dielectric constant measurement as it is easy to be handle and convenient. The measurement of dielectric properties is based on the changes in absorption characteristics of the tuned resonant cavity and resonant frequency shifting [34]. The sample is positioned at a small quartz rod with $0.2 \mathrm{~mm}$ diameter and $\mathrm{h} / 2$ height. Thus, the dimensions of the cavity are only specific to a certain range of frequency while the frequency other than this will required to reconstruct the cavity. Coaxial probe is an alternative of transmission line method. It consists of a coaxial line and tip to sense the reflected signal from the tested material. The tip is usually contact with the substances by immersed in it and operated over a $500 \mathrm{MHz}$ to $110 \mathrm{GHz}$ frequency range [35]. However, it has a limitation as this technique is less efficiency at a very low and high frequencies. Furthermore, the air bubbles which exist in the substances will cause a significant error during measurement. Microstrip structure has more advantages over other techniques as it is smaller in size, cost-effective and require simple fabrication [36]. The most used structure is microstrip line where the samples is placed on the microstrip line and calculate the dielectric properties based on its attenuation. The energy is concentrated within the line and ground plane which also known as fringing field. It is suitable for the low permittivity material measurement with the accuracy better than $5 \%$. Another example for microstrip structure is microstrip resonator where the dielectric properties is measured based on the resonant frequency shifting [37]. According to [38], a microstrip resonator has been presented to measure the dielectric properties of water sample. The measurement was done by means of resonant frequency's variation when NO-3 is added as a pollutant in water sample. Result showed that the proposed microstrip resonator was able to detect the presence of the nitrate with a better performance in resonance enhancing and quality factor. A microstrip patch antenna has also been used as a sensor for dielectric measurement and was be found that it has a better sensitivity compared to microstrip line. It can be used for higher dielectric constant measurement as the sample can be interacted not only at fringing field but also to the near and radiated fields [39]. A work which implement the electronic band gap (EBG) has been presented to measure the industrial waste water and achieve a significant resonant frequency shifting high sensitivity [40]. The result showed that the EBG scheme has enhanced the strength of electric field at the measuring part. The proposed sensor is then tested with various dielectric properties of water samples and can classify the liquids precisely. There are some drawbacks of microstrip structure as it has a low efficiency in dielectric and conductor losses but with a prominent level of cross polarization radiation. Furthermore, the microstrip structure is generally low in power handling capacity and high losses because it is not enclosed, therefore it is susceptible to cross-talk and unintentional radiation.

\section{CONCLUSION}

A review on the water contaminants detection techniques have been done in this paper. The laboratory-based technique was conventionally used and standardized by international organizations, however, the testing cost is relatively high when the number of samples are huge and various type of testing 
are involved. Furthermore, it is time-consuming on transferring the water samples and usually required 2 to 3 days for the analyzed results. The portable testing kits are then been developed to ease and shorten the testing process of lab-based technique. Although this technique is only required for a small amount of water samples, however, the accuracy and precision of portable testing kits are less than lab-based technique. Microwave techniques has been introduced and widely to be used due to its portability, low fabrication cost and robust. As the population growths rapidly, the industrial activities had increased, a new category of emerging contaminants has occurred which is hardly to be detected by conventional techniques. Therefore, an innovative water contaminants detection technique is recommended to be investigate and achieve a fast response at the early stage of warning detection. The designed sensor should be able to classify and quantified the types of the contaminants. Lastly, a continuous and online water quality control system is recommended to be demonstrated to ensure the water quality is always be monitored and monitor the water quality not only in urban areas, but also in rural areas from time to time.

\section{ACKNOWLEDGEMENTS}

The work described in this paper was partially supported by a research grant (Tier-1) by the Office of Research Innovation Commercialization and Consultancy Management (ORICC), Universiti Tun Hussein Onn Malaysia (Grant Ref: H231) and Geran Penyelidikan Pascasiswazah (GPPS), Universiti Tun Hussein Onn Malaysia (Grant Ref: H331).

\section{REFERENCES}

[1] N. H. Ab Razak, S. M. Praveena, A. Z. Aris, and Z. Hashim, "Drinking water studies: A review on heavy metal, application of biomarker and health risk assessment (a special focus in Malaysia)," J. Epidemiol. Glob. Health, vol. 5, no. 4, pp. 297-310, 2015.

[2] K. Tenaga, A. Sekitar, and P. Iklim, "Laporan kualiti alam sekeliling 1," 2017.

[3] N. H. A. Razak, S. M. Praveena, A. Z. Aris, and Z. Hashim, "Quality of Kelantan drinking water and knowledge , attitude and practice among the population of Pasir Mas , Malaysia," Public Health, vol. 131, pp. 103-111, 2015.

[4] W. Yan, J. Li, and X. Bai, "Chemometrics and Intelligent Laboratory Systems Comprehensive assessment and visualized monitoring of urban drinking water quality," Chemom. Intell. Lab. Syst., vol. 155, pp. 26-35, 2016.

[5] O. M. Rodriguez-Narvaez, J. M. Peralta-Hernandez, A. Goonetilleke, and E. R. Bandala, "Treatment technologies for emerging contaminants in water: A review," Chem. Eng. J., vol. 323, pp. 361-380, 2017.

[6] D. Li and S. Liu, "Water Quality Monitoring and Management," 1st ed., p. 362, 2019.

[7] C. De Winter, V. Reddy, D. Worm, and R. Kooij, "Optimal placement of imperfect water quality sensors in water distribution networks," Comput. Chem. Eng., vol. 121, pp. 200-211, 2019.

[8] L. Deshpande, "Water Quality Analysis Laboratory Methods Dispersion \& Dissolution in Water Clouds Water quality Soil and Rocks". 2018.

[9] B. Koki, K. H. Low, H. Juahir, M. Abdul Zali, A. Azid, and S. M. Zain, "Consumption of water from ex-mining ponds in Klang Valley and Melaka, Malaysia: A health risk study," Chemosphere, vol. 195, pp. 641-652, 2018.

[10] K. Tirumalesh, "Simultaneous determination of bromide and nitrate in contaminated waters by ion chromatography using amperometry and absorbance detectors," vol. 74, pp. 1428-1434, 2008.

[11] M. Russel et al., "Development of a detection method based on dielectric spectroscopy for real-time monitoring of meta-cresol contamination in beach-sand," Sensors Actuators, A Phys., vol. 268, pp. 16-26, 2017.

[12] W. Liu and G. Li, "Improve Analysis Precision for ICP-OES and ICP-MS for Environmental and Geological Applications," 2015.

[13] S. C. N. Queiroz, K. Lazou, P. Sandra, and I. C. S. F. Jardim, "Determination of pesticides in water by liquid chromatography-(electrospray ionization)-mass spectrometry (LC-ESI-MS)", vol. 14, pp. 53-60, 2004.

[14] J. S. Dobrowska, S. Erarpat, D. S. Chormey, K. Pryzynska, and S. Bakirdere, "A novel liquid-liquid extraction for the determination of nicotine in tap water, wastewater and saliva at trace levels by GC-MS," pp. 806-812, 2016.

[15] H. M. Albishri, N. A. M. Aldawsari, and D. A. El-Hady, "Ultrasound-assisted temperature-controlled ionic liquid dispersive liquid-phase microextraction combined with reversed-phase liquid chromatography for determination of organophosphorus pesticides in water sample", vol. 19, pp . 2462-2469, 2016.

[16] B. F. L. Lobo and R. Pereiro, "Atomic Absorption Spectrometry: Fundamentals, Instrumentation and Capabilities," Ref. Modul. Chem. Mol. Sci. Chem. Eng., pp. 137-143, 2018.

[17] N. P. Cheremisinoff, "Condensed Encyclopedia of Polymer Engineering Terms," vol. 1, p. 800, 2012.

[18] N. Sakai, Z. F. Mohamad, A. Nasaruddin, S. N. A. Kadir, and A. H. Sulaiman, "Eco-Heart Index as a tool for community-based water quality monitoring and assessment," Ecol. Indic., vol. 91, no. March, pp. 38-46, 2018.

[19] World Health Organization, "Evaluation of the H2S Method for Detection of Fecal Contamination of Drinking Water Water, Sanitation and Health," 2015.

[20] ISO, "Microbiology of food and animal feeding stuffs-Horizontal method for the detection of Escherichia coli O157, ISO 16654:2001," https://www.iso. org/obp/ui/\#iso:std:iso:16654:ed-1:v1:en. 2018.

[21] P. M. Fratamico et al., "Detection by multiplex real-time polymerase chain reaction assays and isolation of Shiga toxin-producing Escherichia coli serogroups O26, O45, O103, O111, O121 and O145 in ground beef," 
Foodborne Pathog. Dis. 8, pp. 601-607, 2011.

[22] R. S. and R. P. F. Soldovieri, L. Crocco, A. Brancaccio, "Applications of ground penetrating radar and microwave tomography in water monitoring and managements," Int. Water Technol. J., vol. I, pp. 73-82, 2011.

[23] O. Korostynska, A. Mason, and A. Al-Shamma'a, "Monitoring of nitrates and phosphates in wastewater: current technologies and further challenges," Int. J. Smart Sens. Intell. Syst., vol. 5, no, pp. 149-176, 2012.

[24] Nasir, S. I. Hussain, B. Soong, and K. Qaraqe, "Energy efficient cooperation in underlay RFID cognitive networks for a water smart home," J. Sensors 14, pp. 18353-18369, 2014.

[25] T. P. Lambrou, C. C. Anastasiou, and M. M. Polycarpou, "A low-cost sensor network for real-time monitoring and contamination detection in drinking water distribution systems," J. Sensors 14, pp. 2765-2772, 2014.

[26] M. Borecki, M. L. Korwin-Pawlowski, M. Beblowska, J. Szmidt, and A. Jakubowsk, "Optoelectronic capillary sensors in microfluidic and point-of-care instrumentation," pp. 3771-3797, 2010.

[27] D. Zengfeng, C. Jing, Y. Wanquan, G. Jinjia, Z. Xin, and Z. Ronger, "Investigation of two novel approaches for detection of Sulfate Ion and Methane dissolved in sediment pore water using Raman Spectroscopy," J. Sensors 15, pp. 12377-12388, 2015.

[28] J. Jiang et al., "Smartphone based portable bacteria pre-concentrating microfluidic sensor and impedance sensing system," pp. 653-659, 2014.

[29] Gross, F. Stangl, K. Hoenes, M. Sift, and M. Hessling, "Improved drinking water disinfection with UVC-LEDs for Escherichia Coli and Bacillus Subtilis utilizing quartz tubes as light guide," pp. 4605-4621, 2015.

[30] X. L. Travassos, S. L. Avila, and N. Ida, "Applied Computing and Informatics Artificial Neural Networks and Machine Learning techniques applied to Ground Penetrating Radar: A review," Appl. Comput. Informatics, pp. 1-7, 2018.

[31] S. F. Senin and R. Hamid, "Ground penetrating radar wave attenuation models for estimation of moisture and chloride content in concrete slab," Constr. Build. Mater., vol. 106, pp. 659-669, 2016.

[32] N. Hafiza, A. Razak, S. M. Praveena, and A. Zaharin, "Drinking water studies: A review on heavy metal, application of biomarker and health risk assessment (a special focus in Malaysia)," J. Epidemiol. Glob. Heal., 2015.

[33] H. Abdelgwad, T. M. Said, and A. M. Gody, "Microwave Detection of Water Pollution in Underground Pipelines," Int. J. Wirel. Microw. Technol., vol. 4, no. 3, pp. 1-15, 2014.

[34] P. Love, J. L., Lanke, E., and Alles, "Estimating the Impact (Energy Emissions and Economics) of the U.S. Fluid Power Industry.," pp. 5-6, 2012.

[35] N. Wagner and T. Sokoll, "Robust low cost open-ended coaxial probe for dielectric spectroscopy in laboratory and in-situ applications," 2011.

[36] M. Bogosanovich, "Microstrip Patch Sensor for Measurement of the Permittivity of Homogeneous Dielectric Materials," IEEE, vol. 49, no. 5, pp. 1144-1148, 2000.

[37] B. Jackson, "A Novel Method for Water Impurity Concentration using Microstrip Resonator Sensor," IEEE International Conference on Recent Advances in Space Technology Services \& Climate Changes RSTS\&CC, November, pp 376-679, 2017.

[38] X. Li and Y. Jiang, "Design of a Cylindrical Cavity Resonator for Measurements of Electrical Properties of Dielectric Materials," Master Programme in Electronics/Telecommunications. 2010.

[39] B. Jackson and T. Jayanthy, "A novel method for water impurity concentration using microstrip resonator sensor," Proc. Int. Conf. "Recent Adv. Sp. Technol. Serv. Clim. Chang. - 2010”, RSTS CC-2010, pp. 376-379, 2010.

[40] F. S. Jafari and J. Ahmadi-shokouh, "International Journal of Electronics and Communications (AEÜ) Industrial liquid characterization enhancement using microwave sensor equipped with electronic band gap structure," AEUE-Int. J. Electron. Commun., vol. 82, pp. 152-159, 2017.

\section{BIOGRAPHIES OF AUTHORS}

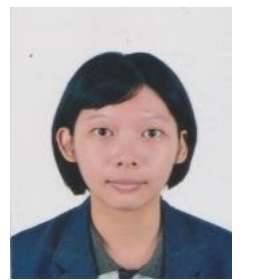

N.T.J Ong: University Tun Hussein Onn Malaysia, 20.1.1994. Josephine Ong is a postgraduate who study in Universiti Tun Hussein Onn Malaysia, 86400 Parit Raja, Batu Pahat Johor, Malaysia. She was graduated from UTHM with a bachelor's degree of Electronic Engineering. She is currently involved in a research project to develop a water quality detecting model by using microwave technique. The developed model should have the ability to identify the type of contaminants and determine its concentration.

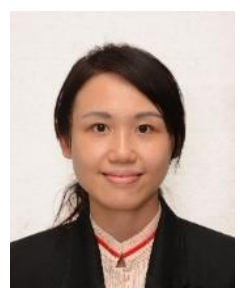

S. K. Yee was born in Johor, Malaysia, in 1983. She received the B.Eng. degree in electrical engineering from Kolej Universiti Tun Hussein Onn Malaysia, Johor, in 2006, and the M.Eng. degree and Ph.D. degree from Universiti Tun Hussein Onn Malaysia (UTHM), Johor, in 2009 and 2015 respectively. Currently she is a senior researcher and lecturer with Research Center for Applied Electromagnetic, Faculty of Electrical and Electronic Engineering, UTHM. Her current research interests include electromagnetic shielding effectiveness and material characterization. 